\title{
THE QUALITY OF MIXING IN MIXERS WITH BARS AND RADIAL AND LONGITUDINAL SCRAPERS
}

Serghei Andrievschi, Associate Professor, PhD., Technical University of Moldova, email: andrievschi.s.p.@mail.ru

Valeriu Lungu, Department Chief., PhD., Technical University of Moldova, Ala Carcea, senior lecturer, Technical University of Moldova, Alexandru Lozan, master, Technical University of Moldova

\section{Abstract}

The process of division - combining of streams in mixers with bars and radial and longitudinal scrapers was studied. The number of unique streams that are formed after passing each row of longitudinal bars and the total amount of them were determined. This is demonstrated by migration of the particles along the mixer from left to right and vice versa, from the center of the drum towards the periphery and vice versa. In the process of mixing the particles in the center gain normal distribution and the ones on the side - sectioned normal distribution. The sum of normal distribution with the sectioned normal distribution leads to an equable distribution along the drum and transverse planes and to a homogeneous mixing of the components. The quality of mixing had been investigated and an optimal mixing regime for the mixer with radial and longitudinal bars and scrapers was proposed.

Keywords: mixer, bar scraper, single stream, distribution, homogeneity

\section{INTRODUCTION}

One of the technological processes with high energy absorption capacity is preparing mixtures used in various fields of national economy: construction, agriculture, automobile industry, food industry, etc.

The existing mixers are equipped with working tools in form of blades, located in different angles towards the direction of their movement. While functioning, the blades form streams of material, inside of whom the mixing does not occur - the entire volume of the material of the blade is pushed forward or forward and lateral. For this reason the energy spending in the mixing process are inefficient; to obtain the required quality of the mixture, the mixing time has to significantly increase.

The intensification of mixing processes, the increase in the degree of homogeneity of the mixture may be obtained not by increasing the consumption 
of energy, but by using a new principle of mixing, using mixing tools in shape of bars. The bars are situated in an optimal way in the space between the shaft and the inner surface of the mixer, which during the rotation provides the forced division of components in a number of streams, their immediate speed combination and the repeatedly repetition of these processes, leading to the migration of such particles along the mixer and the radial direction, in the opposite directions. These processes contribute to a fast mixing of the components with different contents of the mixture.

Between the ends of the mixing tool and the inner surface of the mixer, the blockade of the particles of material may occur. This drawback is removed in the mixer [1] with curved radial bars equipped with elastic longitudinal scrapers, which provide a null rebate between the knife and the inner surface of the drum, while avoiding blocking the particles. The specific energy consumption is lower due to the use of arched bars with an angle less than $90^{\circ}$. The curved bar has a lower resistance when advancing, due to the fact that the maximum value of resistance appears on different portions of its surface, but in case of the bar with an angle of $90^{\circ}$, the maximum resistance occurs simultaneous on the entire length of the bar [2].

This document presents the results of determining the number of single and complex streams while crossing rows of bars through material. The cause of the increase of the mixing process in mixers with bars has been is presented.

The quality of the mixture obtained due to mixing was investigated and an optimal mixing regime was proposed.

\section{RESEARCH METHODOLOGY}

The division of mixture components in streams, the combining of the devised streams and the repetition of these processes were performed graphically, assuming following initial conditions: the size and density of particles are equal for each component, the bars are ideal round and all made of the same material, the division of streams of material is produced in a proportion that is prewritten, streams intersect under a constant angle.

Formed in the process of mixing, the names single stream and complex single stream are used. Each stream, formed separately in the process of splitting and combining, is unique in its own way, because each change of the direction, division in streams, combination of streams lead to the appearance of new single streams and they split, combine further as well. The complex single stream is a stream of particles whose composition includes a number of single streams. 
The investigation of the quality of mixing was performed for two ways of settlement of the mixture components in the drum mixer: in two horizontal layers, one above the other distributed uniformly (Figure 1, $a$ ) and two vertical longitudinal layers evenly placed along the drum (Figure $1, b$ ).

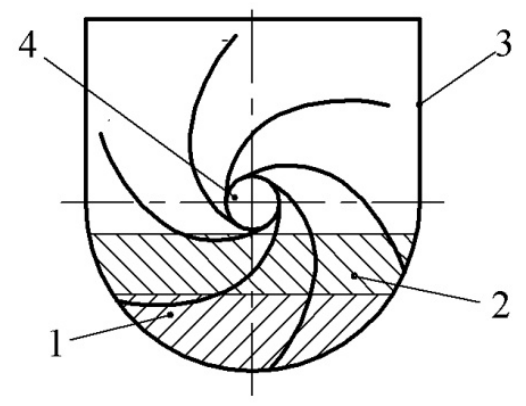

a)

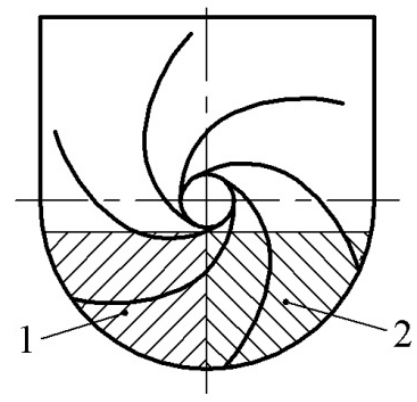

b)

Figure 1. The scheme of the localization of parts:

a) - two horizontal layers, $b$ ) - two layers of vertical longitudinal.

1 - first layer, 2 - the second layer, 3-body, 4 - shaft working bodies

The investigations were carried out using a mixer with an internal diameter of $300 \mathrm{~mm}$ with mixing tools in the form of radial curved bars with a diameter of $10 \mathrm{~mm}$, set in chess on the shaft surface with a longitudinal step of $60 \mathrm{~mm}$ and the angle of $60^{\circ}$ between the longitudinal rows of bar, longitudinal bars, radial rigid and longitudinal elastic scrapers. Minimum number of bars in radial longitudinal rows $n_{b \min }=5$ and a maximum $n_{\text {bmax }}=6$.

The coefficient of filling of the drum was established $K_{u}=0,40$, as a mixture material the particle of size of $a \approx 7 \mathrm{~mm}$ was adopted. For the adopted coefficient of filling, the total volume of particles consists of 10,08 1, with 5,04 1 for each layer of components. The shaft speed $n=60 \mathrm{rev} / \mathrm{min}$. After a certain number of revolutions of the shaft, four samples were taken from four areas of the drum of the mixer (Figure 2). 


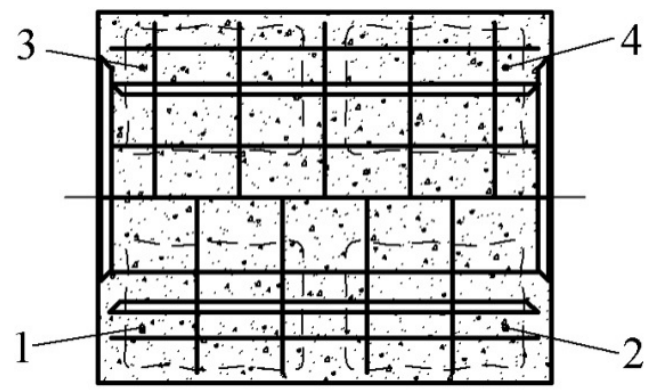

Figure 2. Areas of the drum of the mixer from where samples were taken (top view)

The sample of the mixture of the drum was extracted in a volume of 75 $\mathrm{ml}$. After counting the particles of four samples, the mixture is poured back into the drum. The quality of mixing is determined by the average amount of colored particles (in percent) $\bar{x}$ (average of 16 measurements from four different areas). From each extracted sample the particles were counted, the number of colored particles and the total number of particles were determined.

\section{CONSTRUCTION AND OPERATION OF THE MIXER [1]}

The mixer with arched radial bars and elastic longitudinal scrapers (Figure 3 ) includes a body 1 with semi-cylindrical bottom, mixing tools executed in the shape of arched two arched radial bars 2, fixed radialy on the shaft sections 3, longitudinal bars 4 on radial bars 2, the longitudinal scrapers, attached to the ends of the bars 2 and including plaques 5, the elastic elements 6 and knives 7 are fastened with screws 8 and radial scrapers 9 fixed on the shaft 3, supports 10 , bearings 11 in which the bushings of frontal walls of the body are supported 1 , bearings 12 on which the ends of the shaft are supported 3, a handle 13 attached to the top of the body 1 , shoulders 14 fixed one the exterior surface of the body 1 , leaning on the frame. 


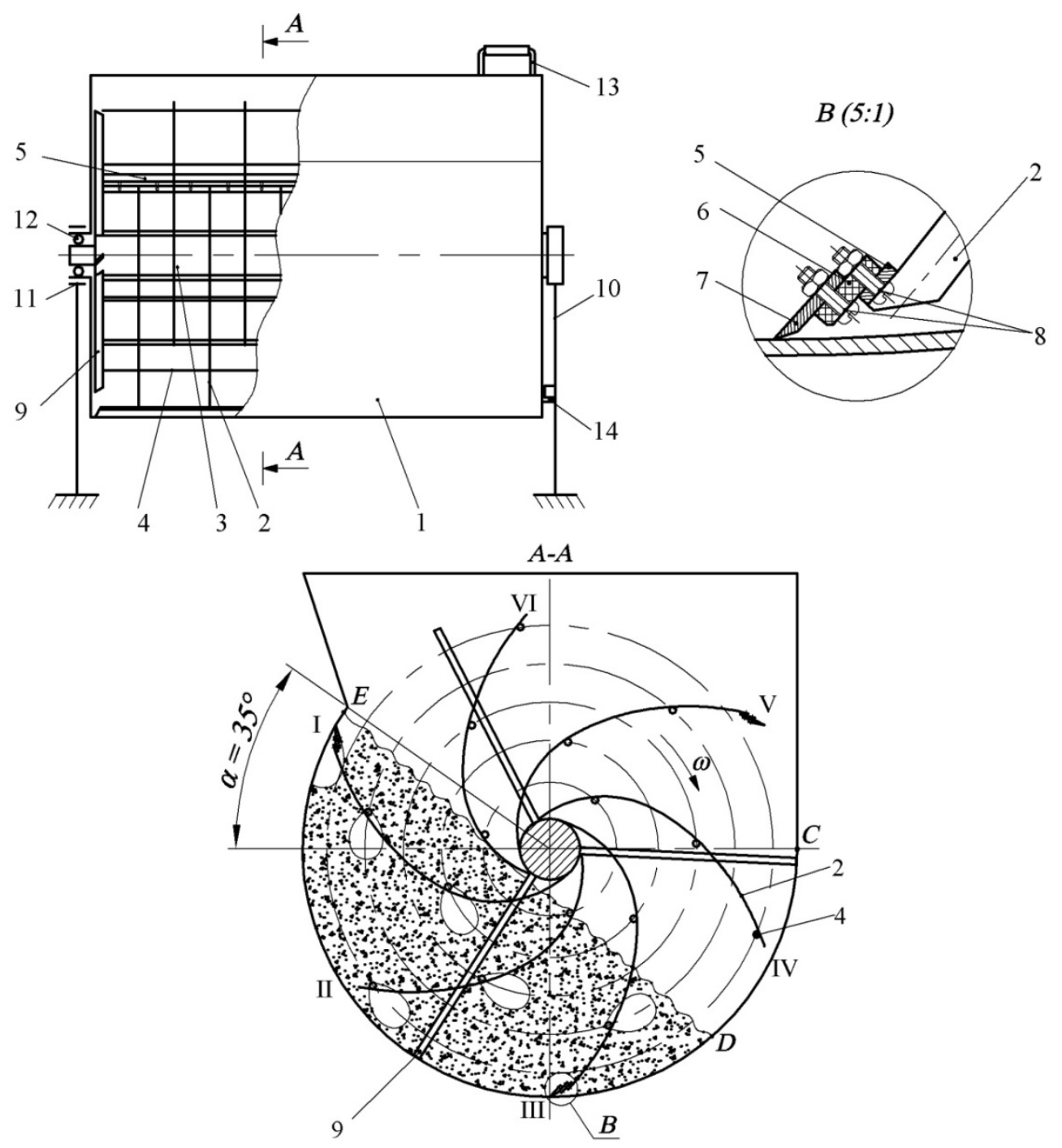

Figure 3. The scheme of the mixer with cyclic action, with arched radial bars and longitudinal scrapers: 1 - body, 2 - radial curved bar, 3 - shaft,

4 - longitudinal scraper, 5 - plaque, 6 - elastic element, 7- knife, 8 - screw; 9 - radial scraper, 10 - support, 11, 12 - ball bearings, 13 - handle, 14 - shoulder

The mixer operates in the following manner. When the shaft is rotating 3 (the driving mechanism is not shown), the material poured into the body 1 , is pierced by radial bars 2, longitudinal bars 4, radial and longitudinal scrapers 9, and split into flows in both longitudinal and transverse planes. Splitting the streams and their combination occur simultaneously in the entire volume of material.

During the operation of the mixer, while the knife 7 of the longitudinal scraper passing through $\mathrm{CD}$ zone (Figure 3 ) due to its own radius that is bigger 
than the inner radius of the drum, the elastic element is bent in the direction of movement of the mixing tool and in this way ensures the slip of the knife 7 on the inner surface of the body 1 . When passing through the DE area, the knife is constantly in contact with the inner surface of the body 1 due to the action of forces of pressing the material in the front. In this way there is provided a void game between the blade tip and the inner surface 7 of the body 1, and as a result - complete avoidance of blocking particles between the blade and the body.

\subsection{The process of mixing in transverse and longitudinal planes}

For describing the mixing process we have to analyze a very limited crosssectional area in the space, for example, the radial bar I zone (Figure 3, to A-A). Radial bars with longitudinal bars fixed on them pass through this area, the material is in static state. This creates the effect of material flow through radial and longitudinal bars, although it is practically in the same place. Only a part of the particles located on the front side of the radial and longitudinal bars and the scrapers can move inside the material for not too large distances, with a certain probability.

For the analysis of the formation of the streams in the transverse plane, we assume that the material is not moving over the drum. When passing the first longitudinal row of bars through material between the scraper and the longitudinal bar, the $\alpha$ flow is formed; between two longitudinal bars - the $\beta$ flow is formed and between the bar from the right and shaft $-\gamma$ flow is formed (Figure 4, $a$, Position 1).

When passing the second longitudinal row of bars in the area, the flows $\alpha$, $\beta$ and $\gamma$ are divided each one in two smaller flows by the longitudinal bars. Since the velocity vector of the small flow from the right $0,5 \alpha$ and the one from left $0,5 \beta$ face each other at an angle, their combining and mixing take place and the flow $0,5 \alpha 0,5 \beta$ results. The same process happens to flow $0,5 \beta$ and $0,5 \gamma$ - the flow $0,5 \beta 0,5 \gamma$ is obtained.

When passing the third row with longitudinal bars through the analyzed zone, the flow $0.5 \alpha$ is targeted to the right of the scraper on the end of the bar. $0,5 \alpha 0,5 \beta$ and $0,5 \beta 0,5 \gamma$ flows are divided by the longitudinal bars into $0,25 \alpha$ $0,25 \beta$ and $0,25 \beta 0,25 \gamma$ flows. The flow $0,5 \alpha$ combines with the flow $0,25 \alpha$ $0,25 \beta$ from left and a new stream $0,75 \alpha 0,25 \beta$ is obtained. The flow $0,25 \alpha$ $0,25 \beta$ from right combines with the flow $0,25 \beta 0,25 \gamma$ from left and the new stream $0,25 \alpha 0,5 \beta 0,25 \gamma$ is obtained. Finally the flow $0,25 \beta 0,25 \gamma$ combines with the flow $0,5 \gamma$ and the new stream $0,25 \beta 0,75 \gamma$ is obtained. 

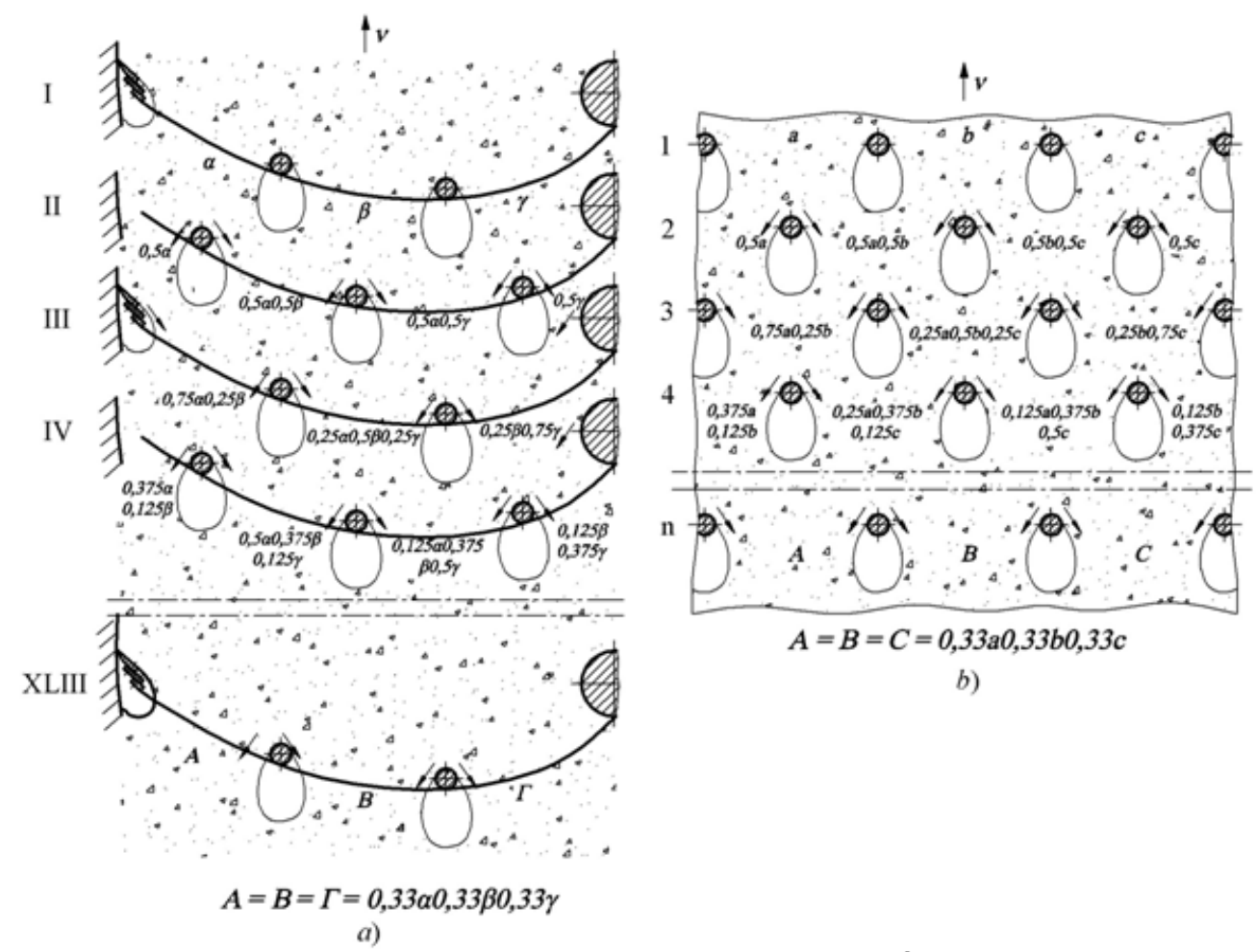

b)

Figure 4. Scheme of the process of splitting - combining the streams: $a$ ) in the transverse plane, $b$ ) in the longitudinal plane

We observe (Figure 4, a) that part of the material of the flow $\alpha$ in the process of division-combining gradually moves on the right side of the area and when passing the fifth row of longitudinal bars, it arrives at the shaft and then stars moving on the left. Also some of the material of the flow $\beta$ gradually moves to the left and reaches the mixer body when passing the fifth row through the analyzed area and then starts migrating to the right. The material of the flow $\gamma$ in the process of splitting-combining moves to left and right, reaching the mixer body until the shaft, and then starts moving in opposite directions.

At the beginning of mixing process, the content of flows $\alpha \beta$ and $\gamma$ in newly formed streams is not uniform. Gradually this unevenness disappears, and when passing through the study area of the thirteenth row of longitudinal bars (starting with the first one), flows $\mathrm{A}, \mathrm{B}, \Gamma$ are obtained, which contain particles uniformly distributed from initial flows $\alpha, \beta$ and $\gamma$ (Figure 4, a). The migration process of particles of the flows takes place not only in the analyzed zone, but in the whole transverse section of the drum. This contributes to the rapid mixing of the material in the transverse plane, and if we consider all sections - all volume in the transverse planes of the drum. 
The process of mixing in the longitudinal plane is similar to the one described above and shown schematically in Figure $4, b$. Because of the flow division-combination, the particles of the material migrate simultaneously from left to right and vice versa. The division, combination and migration processes take place simultaneously in all areas of longitudinal rows of bars located in the material, leading to an intensive and homogeneous mixing of the mixture components in its entire volume.

\section{THE STRUCTURE OF STREAMS}

The mixing process described above was characterized by the number of streams of complex quantitative and their amount in the composition of the original streams.

The number of complex streams formed in the process of mixing can be determined graphically shown in the figure 5, in which there is indicated the number of longitudinal rows of bars $n_{r}$, the location of the bars on the surface of the shaft, the number of complex streams $n_{s c}$ and the total number of complex streams $\sum n_{\text {şc }}$.

The docket number of complex streams that is formed after each longitudinal line of bars represents an arithmetic progression whose difference is equal to ten (Figure 5, a). The same regularities are observed in dividing components in transverse planes (Figure 5,b).

After the passage of 18 rows of bars (which corresponds to three revolutions of the shaft) 252 of complex streams are formed. After 18 turns (18 seconds), going through the material of 108 rows of longitudinal bars, the total number of complex streams is 432 in transverse planes and 1080 in longitudinal planes, 1512 complex streams in total.

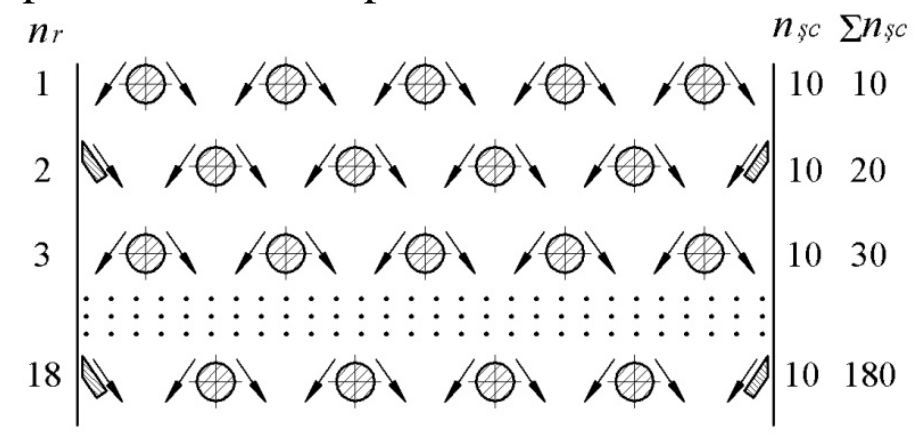

a)

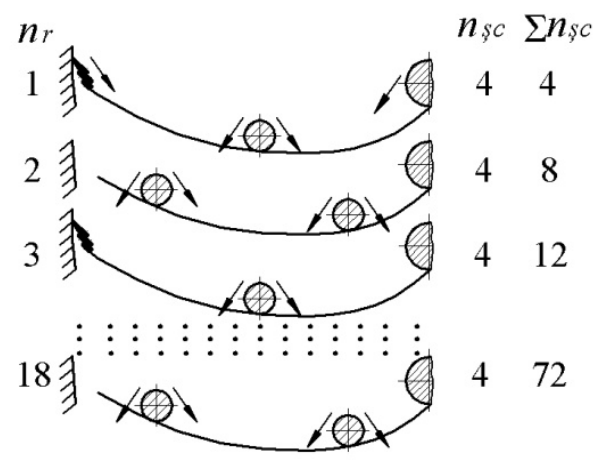

b)

Figure 5. The scheme of division of the material in the streams and subsequently combining them to longitudinal planes $(a)$ and transverse plane $(b)$ 
These streams represent the macrostructure of the prepared mixture. The composition of each complex stream is obtained from the dividing result, combining bars and diverting scrapers.

The total number of unique streams which are formed in the mixing progress is shown in the Figure 6. The initial amount of the material is equal to one unit, so at the first longitudinal row of bars 10 unique streams will be formed with each quantity equal to 0,1. In Figure 6 the process of formation of unique streams to other longitudinal rows of bars are shown. The stream divert of the scraper changes its combination. That's why new unique streams appear at scrapers too.

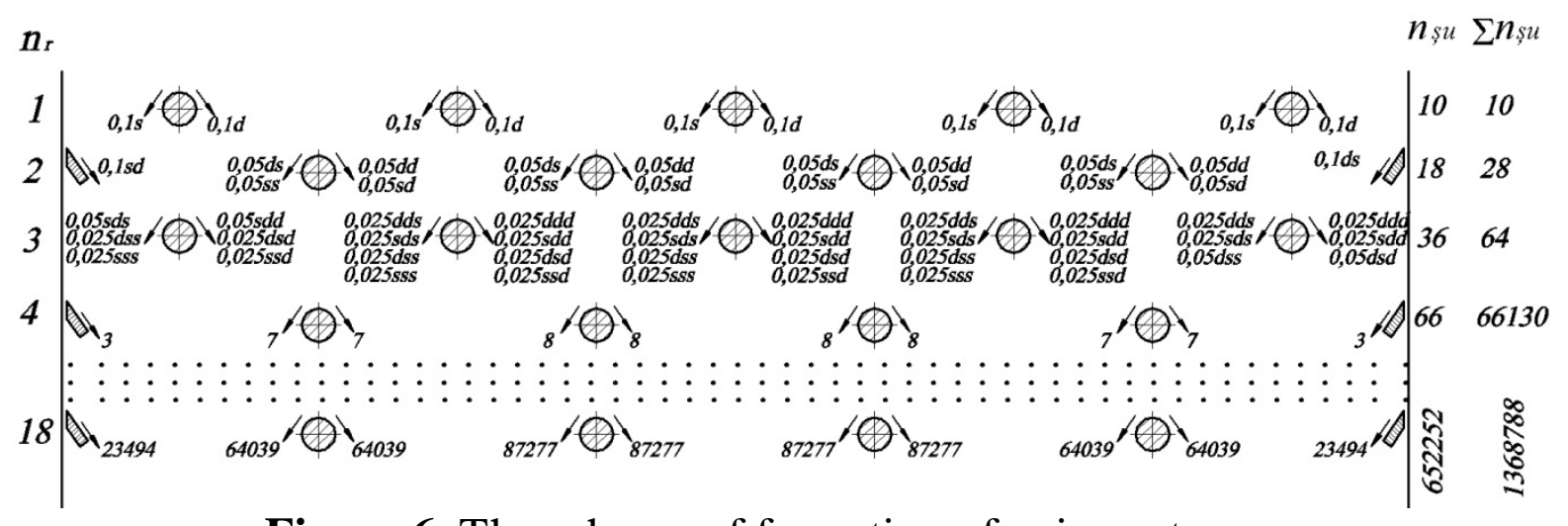

Figure 6. The scheme of formation of unique streams

The mixing process shown in the Figure 6 demonstrates that the microstructure of the complex stream meets a large number of unique streams.

After passing the 18 rows of longitudinal bars through the material, the total number of unique streams is 1.368 .788 , and in transverse plane 135.588. These streams are mixed together; the particles migrate along the mixer as well as transversely in opposite directions.

\section{THE EXPERIMENTAL RESULTS}

The results of both studied cases are shown in the Figure 7. The mean values were calculated $\bar{x}_{1}, \bar{x}_{2}, \bar{x}_{3}$ and $\bar{x}_{4}$ the amount of colored particles from the same for each area of taking the sample and mean value $\bar{x}_{m}$ which characterize the homogeneity of the whole mixture. 
ROMANIAN JOURNAL

OF TRANSPORT INFRASTRUCTURE

Serghei Andrievschi, Valeriu Lungu, Ala Carcea, Alexandru Lozan,

The quality of mixing in mixers with bars and radial and longitudinal scrapers

a)

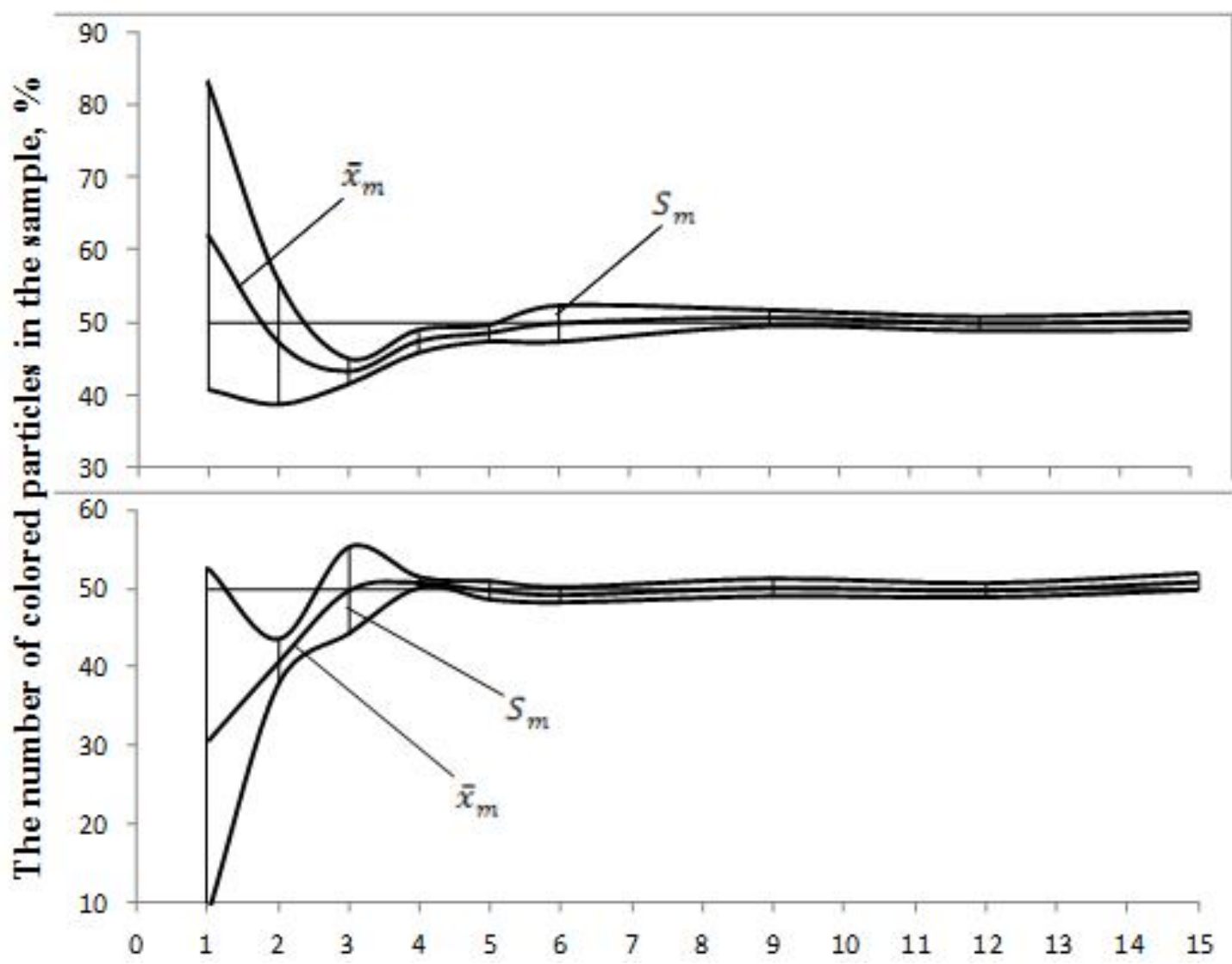

Figure 7. Dependence of average and mean square deviation $S_{m}$ of the number of turns of shaft mixer for the case of locating the mixture components in the drum mixer in two horizontal layers $-a$ ), and in two longitudinal layers $-b$ )

The mean square deviation $S_{1}, S_{2}, S_{3}, S_{4}$ and $S_{m}$ of the average values has been calculated. We see that the experimental results are characterized by the small quadratic deviation and this explains the fact that the homogeneity of the mixture in zones 1 and 2, 3 and 4 is almost the same.

The results describe adequately the mixing process, at first the heterogeneous of mixture decreases sharply to achieve the maximum homogeneity that is constant in the continuation of further mixing.

From the diagram (Fig. 7, a) we observe that for the initial of locating the mixture components in two horizontal layers one above another, the mixture became homogeneous after 6-th rotation of the mixer shaft, because the mean value which characterized the homogeneity in the entire mixture is equal to $49,88 \%$ and the standard deviation $S_{m}$ is equal to $2,47 \%$, but the increase of the quality of the mixture takes place until the 9th rotation, the mean square deviation $S_{m}$ decreasing to $1,08 \%$. Minimizing and the broadly constant at the 
value of the average coefficient of variation $v$ took place after the 9th rotation $(v=2,14 \%)$.

For the case of initial locating of the mixture components in two vertical longitudinal layers, the mixture becomes homogenous after the 4-th rotation (Figure 7,b) the average being 50,74\% and the mean square deviation $S_{m}=0,68$. Continuing the mixing process until the 15-th rotation, the quality of the mixture remain unchanged, which means that the maximum quality of the mixture was obtained after the 4-th rotation. The average of the coefficient of variation for this case is still minimal after the 4th rotation and is $1,34 \%$ but at continuing mixing it remains almost unchanged.

\section{CONCLUSIONS}

1. In the mixer with radial and longitudinal bars and radial and longitudinal scrapers, there is a complex mixing process that leads to the formation of a very large number of single streams, the migration of particles along the mixer and across in both directions.

2. Using the method of assessing the quality of the mixture, when a layer is composed solely of colored particles and the other layer - of not colored particles, allows to obtain some exact experimental results, which can be used to assess the quality of the mixture changes depending on the number of revolutions of the mixer shaft.

3. In case of the initial settlement of the mixture components in the mixer drum in two horizontal layers one above another, the high quality of the mixture was obtained after six turns of the shaft; and in case of the components settlement in two vertical longitudinal layers - after four revolutions of the shaft mixer, due to the specific position of the radial and longitudinal bars in the space between the shaft and the drum. The mixing regime is proposed as following: 6 seconds while dry, 6-12 seconds while wet.

\section{REFERENCES}

[1]. ANDRIEVSCHI SERGHEI., LOZAN ALEXANDR. Mixer with cyclic action. Positive decision nr.7356 from 2012.11.12 by AGEPI MD.

[2]. ANDRIEVSCHI SERGHEI. Intensificarea procesului de amestecare în malaxoarele cu organe de lucru în formă de bare. Univ. Tehn. a Moldovei. - Ch.: UTM, 2008, 176p. ISBN 978-9975-45-088-1. 